\title{
Genomes against parasites
}

Whereas once it was only possible to generate reference genome sequences one at a time, it is now feasible to design genomic experiments to maximize functional comparisons and contrasts among many genomes. Investigating the diversity of a number of related parasite genomes and their transcriptomes in multiple contexts increasingly shows us ways to intervene in the ecological and evolutionary strategies of pathogens.

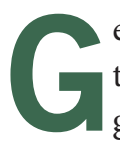
enetics is at its heart a method that compares the contrasting phenotypes of differing genotypes. Wholegenome sequencing in isolation is essentially descriptive, providing the data to address an almost infinite number of hypotheses, but it may not yield much functional information unless the right genomes and phenotypes are available to make the appropriate comparisons. Without wanting to detract from the (still remarkable) achievement of sequencing, assembling and annotating genomes, we note our view that the choice of source (geographic location, phenotype and time) of experimental material is enormously important. Although it is now possible to sequence large numbers of genomes in parallel, appropriate sampling of the within-species variation is an essential step upon which the resulting conclusions depend. Understanding the scale of variation may require many iterations of sequencing, sampling and resequencing.

Initially, most authors compare their newly generated reference genome to extant genomic data, starting with betweenspecies comparisons and phylogeny reconstruction, and then search for regions with evidence of horizontal transfer. In the case of pathogen genomics, we are usually looking for interventions into parasite biology that can be made immediately; thus, whole-genome analysis at the population level can be a very effective experimental strategy.

In this issue, Kathryn Holt and colleagues (p. 1056, News \& Views p. 964) use this approach to identify a small subset of Shigella sonnei strains responsible for much of the burden of dysentery worldwide that have recently spread globally and have acquired antibiotic resistance.
The diversity of eukaryotic parasite genomes is also investigated herein (p. 1046, News \& Views p. 962). Daniel Neafsey and colleagues report that a set of monkey-grown Plasmodium vivax genomes exhibit approximately twice the SNP diversity of a similar geographic sampling of Plasmodium falciparum cultures. In a complementary paper, Shin-Ichiro Tachibana and colleagues (p. 1051) sequenced three Plasmodium cynomolgi genomes to round out our understanding of the closest relatives of P. vivax. These sequences will be invaluable for the systematic investigation of host-parasite interactions in the malaria infections of human and nonhuman primates.

Human health ultimately depends on the security of the food supply; thus, it would be a disaster to neglect the evolutionary struggle between agricultural plants and their parasites. Richard O'Connell and colleagues herein report (p. 1060) the genomes and transcriptomes of two Colletotrichum fungal pathogens, one infecting a range of dicot plants and the other more specific for maize, a monocot. These fungi produce specialized hyphal types during their life cycles that enable them to pursue twin ecological strategies: living within growing plant cells and upon the decaying plant. Comparison of in vitro-grown fungi with those growing on host plants gave transcriptome-based clues to the sensory function of invasive fungal organs-appressoria-that are key to these strategies and that are here shown to be capable of responding to host signals during in vivo infection.

We welcome these diverse approaches to genomic analysis of parasites and are confident that genomic methods, guided by carefully chosen comparisons, will maximize the yield of new biological insights. 